\title{
Modification of wavelet transform approach for low-wind direction extraction
}

\begin{abstract}
Regional wind possession that experienced low wind study gives challenged the researcher in recognizing high spatial behavior of wind. Current practices of wind direction retrieval involved the derivation of wavelet coefficient through wavelet decomposition and wavelet transform in Fourier window of satellite images. However, mentioned technique underestimate wind streak of low wind speed where most of the studies have focueds only at high wind speed area. This study aims to enhance wavelet decomposition through enhancement of multi resolution analysis. The images used in this study covered low wind speed area $(3-5 \mathrm{~m} / \mathrm{s})$ and consist of two modes of RADARSAT-1 SAR images; Standard-2 and Wide-3 modes. Identification of the most suitable modes for low-wind direction extraction signifies the use of two types of modes. Fast Fourier Transform function was applied on wavelet coefficient window of RADARSAT-1 SAR data. The extension work was validated using Quikscat wind direction data and it agrees with the method with r-squared values of 0.6233 for Standard-2 and 0.6483 for Wide- 3 images. The extension of the study has potential application in the extraction of wind direction in low wind speed area.
\end{abstract}

Keyword: Fast-fourier transform (FFT); Low-wind speed; Multi-resolution analysis; RADARSAT-1 SAR; Wind direction 